\title{
Comparison of Two Formulations of Sodium Divalproate Plasma Concentrations after a Single 500 mg Oral Dose in Healthy Subjects, and Stochastic Sub-analysis of the Individual "Clinical Perceptible" Levels
}

\section{Nunez DA*, Schiaffino S and Roldán EJA}

Scientific Direction Gador SA, Buenos Aires, Argentina

\begin{abstract}
A new generic formula containing $500 \mathrm{mg}$ of sodium divalproate in enteric releasing coated tablets was compared to the reference product in a pharmacokinetic, randomized, cross-over $2 \times 2$ study performed in 24 healthy adult males. After administering a single dose, it was demonstrated that the maximum plasma concentration of valproic acid varies within the $98.4-113.0$ range for $90 \% \mathrm{Cl}$ (test/reference); and the area under the curve of plasma levels over the $24 \mathrm{~h}$ period following the intake varies within the $91.1-99.5$ range; therefore, it can be seen that both parameters are within the range of acceptance for the interchangeability of the products. Individual results show the typical inter- subject and inter -treatment variations of divalproate. Since the latter have an impact on the prescription decision, results are sub-analyzed considering a plasma threshold of valproic acid as effective or stochastically clinical perceptible $\left(\mathrm{C}_{\text {per }}\right)$. Complete duration of plasma levels above $\mathrm{C}_{\text {per }}$ is comparable for both formulations $(p<0.81)$; nevertheless, the test product reaches the $C$, value faster than the reference compound $(p<0.04)$. Therefore, we consider that the individual perception of efficacy of the generic product should not be inferior to that of the reference. In conclusion, the tested formula is bioequivalent and the further sub-analysis of individual variations above a plasma level of stochastic clinical perception of effects shows non-relevant differences between both brands.
\end{abstract}

Keywords: Divalproate; Bioequivalence; Generic; Formulation quality

\section{Introduction}

The long-term result in the treatment of patients with epilepsy depends on the appropriate selection of the therapeutic regimen, the optimum individual dose, strict patient adherence to treatment and close monitoring of efficacy and safety.

To reach the aim successfully, the use of appropriate antiepileptic drugs and their supply by means of quality pharmaceutical formulations are important aspects of the therapeutic decision. Valproic acid has been widely preferred over the last decade $[1,2]$. The product is yet indicated for migraine crisis prophylaxis [3]. Subsequent findings have suggested its usefulness in ictal-like manifestations, including anxiety disorders, mental problems related to chronic alcohol abuse, and certain mood disorders $[4,5]$. In these widely different conditions, however, the relationship between the clinical perception of efficacy and plasma levels has not shown to be strictly linear and it is necessary to set an appropriate individual dose in several patients.

In addition, sodium divalproate is an equal proportion combination of sodium valproate and valproic acid, which is dissociated in the gastrointestinal tract giving origin to the active principle, ionic valproate. One of the main characteristics of valproic acid is having a dose-limited absorption, a non-linear plasma protein binding kinetics, and multiple metabolic elimination routes [6]; aspects that favor objective variations in individual responses.

Nowadays, sodium divalproate is manufactured and marketed by different pharmaceutical companies; nevertheless, product interchangeability may be concerned in practice due to eventual differences observed in the clinical results after the interchange among certain branded or non-branded products $[7,8]$. For some indications, these differences may be noticed objectively or subjectively as we mentioned before, probably both. However, generic formulations play an important role in the accessibility of the drug to huge population sectors, especially because they optimized the cost-benefit relationship in favor of the patient, his/her family group and social security insurance. Therefore, by analyzing the expected individual variability range between two formulations with bioequivalent statistical parameters is a valuable piece of information for the therapist who judges recommending interchangeability to his/her patients beneficial. And with more interest will be to observe these variations not in the whole curve under the plasma levels, which are bioequivalent in marketed products, but in the part of the curve which can be considered with more impact in effects, that is the duration time of the higher levels within the interval between doses.

Indeed, the whole area under plasma levels does not strictly relate to the concentrations clinically expressing effects, which in turn, clinical perception appears above some critical levels only, may differ from patient to patient, and may be perceived differently by practitioners. And it's mainly the latter fact what in practice decides to interchange or not a formulation. Therefore it is interesting to further analyze the individual behavior of the formulations and thus produce supplementary data. With that objective in mind, here we report the results of a formal regulatory bioequivalence study comparing a new oral formulation of divalproate (Divalprex from Gador SA, Buenos Aires) with the reference product according to section 3 of ANMAT Provision No. 2807/02 (Valcote" from Laboratorios Abbott Argentina). The trial statistically showed the interchangeability between both

*Corresponding author: Nunez DA, Scientific Direction Gador SA, Buenos Aires Argentina, E-mail: cproverbio@gador.com.ar

Received June 25, 2013; Accepted August 28, 2013; Published September 04 2013

Citation: Nunez DA, Schiaffino S, Roldán EJA (2013) Comparison of Two Formulations of Sodium Divalproate Plasma Concentrations after a Single 500 mg Oral Dose in Healthy Subjects, and Stochastic Sub-analysis of the Individual "Clinical Perceptible" Levels. J Bioequiv Availab 5: 197-200. doi:10.4172/ jbb.1000158

Copyright: @ 2013 Nunez DA, et al. This is an open-access article distributed under the terms of the Creative Commons Attribution License, which permits unrestricted use, distribution, and reproduction in any medium, provided the original author and source are credited. 
products within acceptable confidence margins. From a subsequent analysis, individual data are further described so as to reach predicted valuable conclusions for the practical therapist.

\section{Methods}

\section{Subjects}

This study recruited male volunteers, aged 21 to 40 years, with a Quetelet index within 20 to $27 \mathrm{~kg} / \mathrm{m}^{2}$. According to the evaluation performed by the main investigator, all subjects were in healthy conditions. The investigator considered the subjects healthy if the clinical analysis and radiological, cardiological and clinical exams had normal results or showed no clinical relevance. The same investigator also evaluated the subject as reliable taking into account as a criterion the participation of the subject in previous studies. The volunteer had to show responsibility, honesty, compliance and understanding of what his/her participation in the study involved. Some of the inclusion criteria were having no significant alterations in run-in studies, being non-smoker and non-drinker, having signed the informed consent, having completed any drug therapy at least two weeks before the beginning of the study. Exclusion criteria were receiving any chronic drug therapy, having an addiction, having participated in another clinical study within the previous three months, need to start any treatment during the period of this study, which was inconsistent with this study, donation or blood loss of $450 \mathrm{~mL}$ or more in the last three months, evidence or history of diabetes, cardiovascular, pulmonary, renal, hepatic, pancreatic disorders, and disorders of the urea cycle, and hematological, metabolic, dermatological, gastrointestinal, immune disorders, endocrine or neoplastic diseases, hypersensitivity to drugs or excipients, condition or history of psychiatric illness, epilepsy or seizures.

The whole study was conducted in compliance with the registration requirements under the Good Clinical Practice stated in applicable ANMAT Provisions No. 6677/10, No. 3185/99 and supplementary regulations.

\section{Formulations}

The test formulation was an enteric releasing coated tablet containing $538.1 \mathrm{mg}$ of sodium divalproate, equivalent to $500 \mathrm{mg}$ of valproic acid, Divalprex, GADOR S.A., Buenos Aires (batch 07766), and the reference product was a similar tablet, Valcote, Abott Laboratories (batch $86164 \mathrm{QA}$ ).

\section{Study design}

The clinical trial was conducted with a cross-over $2 \times 2$ design, with randomized groups of treatments. Both pharmaceutical forms were administered within 30 minutes after breakfast. During the whole experimental day the food consumption was of 2918 calories. Washout period was of 14 days, i.e., an interval greater than 5 half-lives of the active principle.

\section{Analytical methodology}

Valproic acid was quantified in plasma using a validated method. The HPLC method consisted in the extraction of plasma valproic acid using as internal standard cycle-pentane-carboxylic acid (CPCA) from acidified plasma with $\mathrm{H}_{3} \mathrm{PO}_{4}$ in a mixture of n-hexane and t-butylmethyl-ether. An aliquot of the organic phase was treated with a suspension of potassium carbonate and 18-crown- 6 potassium ether to transform it in a non-volatile salt. Solvents evaporate till dryness and the residue was treated with bromo-methyl-dimethoxy-coumarin in presence of $\mathrm{K}_{2} \mathrm{CO}_{3}$ and 19-crown-6 potassium ether for the subsequent analysis using reverse phase HPLC and fluorescence detection. The method allowed for the selective determination of valproic acid in a range within 1.2 to $240.0 \mu \mathrm{g} / \mathrm{mL}$ from only $100 \mu \mathrm{L}$ of plasma.

\section{Pharmacokinetic calculations}

The pharmacokinetic and biostatistics analysis was performed using Win Non Lin, v 5.3 professional from Pharsight (St. Louis, USA). Pharmacokinetic parameters were obtained from blood concentration curves of the drug versus time and were statistically analyzed for bioequivalence determination. From a total of 32 evaluated candidates, 28 were selected to participate in the bioequivalence study considering $\mathrm{n}=24$ plus 4 volunteers as extra patients; out of these, 27 effectively entered the clinical phase, since one volunteer did not enter the study. Out of the 27 volunteers that finally entered the clinical phase, only the results of 24 of them were used in the statistical phase according to the protocol, with no need to include the 3 additional participants in the analysis.

The following pharmacokinetic parameters for sodium divalproate were determined: $\mathrm{C}_{\max }(\mu \mathrm{g} / \mathrm{ml})$ or maximum plasma valproic acid concentration, $\mathrm{T}_{\max }(\mathrm{h})$ or time to reach the maximum value of plasma valproic acid, $\mathrm{t}^{\mathrm{t} / 2} \mathrm{max}$ (h) or apparent time of plasma valproic acid half-life, $\mathrm{AUC}_{\text {last }}(\mu \mathrm{g} . \mathrm{h} / \mathrm{ml})$ or area under the curve of plasma valproic acid since 0 hour to last hour of determinations $(24 \mathrm{~h})$ and $\mathrm{AUC}_{\text {tot }}(\mu \mathrm{g} . \mathrm{h} / \mathrm{ml})$ or area under the curve of plasma valproic acid from time 0 extrapolated to infinite.

The following bioequivalence criterion was established in the protocol: The $90 \%$ confidence interval $(90 \% \mathrm{CI})$ of $\mathrm{C}_{\max }$ Test/ $\mathrm{C}_{\max }$ Reference and $\mathrm{AUC}_{\text {last }}$ Test/ $\mathrm{AUC}_{\text {last }}$. Reference ratios should be within the $80-125 \%$ range of acceptability. Furthermore, the $\mathrm{AUC}_{\text {last }}$ parameter should not be less than the $80 \%$ of the $\mathrm{AUC}_{\text {tot }}$ parameter.

For the $\mathrm{C}_{\max }$ y AUC pharmacokinetic parameters, the estimated point $(\mathrm{T} / \mathrm{R})$ and the $90 \% \mathrm{CI}$ were calculated for the ratio between treatment means, previous logarithmic transformation of plasma valproic acid concentrations using the mixed models tool and considering "treatment" as fixed effect and "subject" as random effect. The ANOVA method was used when analyzing the effect of each variation factor and calculating the variability with the mean square error or residual variance corresponding to the model.

\section{Sub-analysis of treatment data in same individuals}

After the formal bioequivalence study, sample dada were analyzed differently to give the study an additional clinical connotation. The 20.0 $\mu \mathrm{g} / \mathrm{mL}$ value of plasma valproic acid was stochastically chosen as a given effective plasma concentration $\left(\mathrm{C}_{\text {per }}\right)$ after the intake of $500 \mathrm{mg}$ of the active ingredient and according to figures published by other authors [9-11]. During the 24 hour period after the intake of the test or reference formulation, the duration of plasma valproic acid levels above $\mathrm{C}_{\text {per }}$ in each subject $\left(\mathrm{T}_{\text {per }}\right)$, the time to achieve $\mathrm{C}_{\text {per }}$ plasma levels after the intake of the drug, and the time in which plasma concentrations decay below $\mathrm{C}_{\text {per }}$ were determined. These variables were compared using 2-tailed test- $T$ for independent groups and different variances. The number of subjects whose $\mathrm{T}_{\text {per }}$ differences exceeded 2 hours intra-individually after the intake of both study formulations were estimated as an indication of possible variations that may be clinically (subjectively or objectively) perceptible. 
Citation: Nunez DA, Schiaffino S, Roldán EJA (2013) Comparison of Two Formulations of Sodium Divalproate Plasma Concentrations after a Single $500 \mathrm{mg}$ Oral Dose in Healthy Subjects, and Stochastic Sub-analysis of the Individual "Clinical Perceptible" Levels. J Bioequiv Availab 5 : 197-200. doi:10.4172/jbb.1000158

\section{Report of adverse events}

Events were reported in compliance with applicable ANMAT Provision No. (1067/08), which defines them as serious or non-serious, and then according to their defined, probable, possible or unrelated relationship with the trial medication. Since the sample size does not have enough statistical power, cases are only reported as received from the research unit without statistical estimation.

\section{Results}

The main anthropo-morphometric data of the sample under study are summarized in Table 1.

\section{Data analysis}

Based on plasma concentration results obtained in the 24 participants (Figure 1), pharmacokinetic parameters were estimated for sodium divalproate (Table 2). During the statistical analysis and in compliance with the good practices, no residual effect was detected, there were no deviations from actual extraction times, plasma concentrations detected were within the linearity range, there were neither dropouts nor outliers, and only $1.9 \%$ of the samples were reanalyzed. Results from both $\mathrm{AUC}$ and $\mathrm{C}_{\text {max }}$ estimates are similar, with $\mathrm{AUC}_{\text {last }}$ being above $90 \%$ of the $\mathrm{AUC}_{\text {tot }}$ in all cases. Also, a higher dispersion of $\mathrm{T}_{\max }$ can be seen with the test drug, prolonging the average value of the group. This variable, however, was not predefined as an endpoint.

Bioequivalence results are shown in Table 3. All endpoints have proved bioequivalence and acceptability for the interchangeability of both formulas within the $80-125$ range for $90 \%$ CI.

Figure 1 shows divalproate mean plasma levels duration in function of time after the intake of a single dose of the test and reference products. Table 4 summarizes the subsequent analysis and shows statistical comparisons of $\mathrm{T}_{\text {Cper }}$. In subjects taking both formulations in

\begin{tabular}{|l|l|l|l|}
\hline & Mean & Min & Max \\
\hline Age (years) & 27.6 & 21 & 38 \\
\hline Body weight $(\mathrm{Kg})$ & 75.8 & 62 & 90 \\
\hline Height $(\mathrm{cm})$ & 177.5 & 165 & 193 \\
\hline BMI & 23.8 & 20 & 27 \\
\hline
\end{tabular}

Table 1: Main features of the sample included in the pharmacokinetic study of two formulations of sodium divalproate ( $n=24$ men).

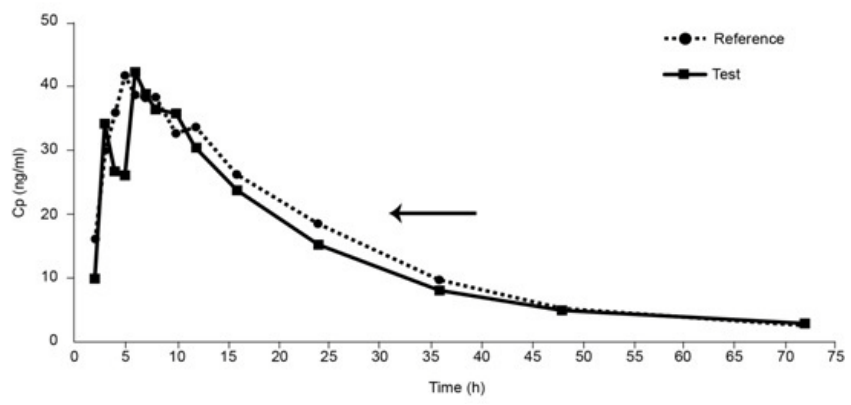

Figure 1: Valproic acid plasma levels $(\mathrm{Cp})$ in function of time after the intake of a single $500 \mathrm{mg}$ dose of sodium divalproate enteric coated tablets of a test (square) and a reference (circle) product by 24 healthy men in a crossover design. The arrow marks the Cef or valproic acid plasma concentration considered effective for the further intra- individual comparison (see text and Figure 2).

\begin{tabular}{|c|c|c|c|c|c|}
\hline Proc & $\underset{(\mu g / m L)}{C_{\max }}$ & $\mathrm{T}_{\max }(\mathrm{h})$ & $T^{1 / 2}(h)$ & $\underset{(\mu \mathrm{g} \cdot \mathrm{h} / \mathrm{mL})}{\mathrm{AUC}_{\text {ult }}}$ & $\underset{(\mu \mathrm{g} \cdot \mathrm{h} / \mathrm{mL})}{\mathrm{AUC}_{\text {tot }}}$ \\
\hline Test & $46.5 \pm 6.8$ & $6.3 \pm 1.9$ & & $853.4 \pm 177.2$ & $923.9 \pm 215.4$ \\
\hline Reference & $44.2 \pm 6.7$ & $9.6 \pm 5.9$ & $15.3 \pm 4.2$ & $902.1 \pm 213.5$ & $969.3 \pm 246.0$ \\
\hline
\end{tabular}

Table 2: Peak plasma valproic acid levels $\left(C_{\max }\right)$, time to achieve $C_{\max }\left(T_{\max }\right)$, plasma elimination half-life $\left(T^{1} / 2\right)$, and area under the curve $0-24$ hrs ( $A \cup C_{w t}$ ) and extrapolated to infinite $\left(A \cup C_{\text {tot }}\right)$, after the intake of two enteric coated formulations (test and reference) containing each one $500 \mathrm{mg}$ of sodium divalproate, single dose, by 24 healthy men.Figures are mean and SD.

\section{Test/Reference}

\begin{tabular}{|l|l|l|l|l|}
\hline & Geometric Mean & Aritmetic Mean & Cl 90\% & Power \\
\hline$C_{\max }$ & 1.05 & 1.05 & $98.4-113.0$ & 0.9995 \\
\hline AUC $_{\text {ult }}$ & 0.95 & 0.95 & $91.1-99.5$ & 1.0000 \\
\hline
\end{tabular}

Table 3: Bioequivalence analysis of plasma valproic acid maximum plasma concentration $\left(C_{\max }\right)$, and the area under the curve during the 0-24 hr period $\left(\mathrm{AUC}_{\mathrm{ut}}\right)$, or extrapolated to infinitum $\left(\mathrm{AUC}_{\mathrm{tot}}\right)$, calculated with $\mathrm{Cl} 90 \%$ and statistical power above $99 \%$. All the ranges are within the $80-125 \%$ range of acceptance for the interchangeability of the products.

\begin{tabular}{|l|c|c|c|c|c|c|c|}
\hline & $\mathbf{n}$ & $\begin{array}{c}\text { Time to } \\
\text { achieve } \\
\text { Cper }\end{array}$ & Dif.T/R & $\begin{array}{c}\text { Time to } \\
\text { decay } \\
\text { below Cper }\end{array}$ & Dif.T/R & $\mathbf{T}_{\mathrm{em}}$ & Dif.T/R \\
\hline Test & 24 & $6.1 \pm 2.2$ & $-2.6 \pm 4.7$ & $15.6 \pm 3.2$ & $-2.3 \pm 4.6$ & $9.5 \pm 3.0$ & $0.25 \pm 2.1$ \\
\hline reference & 24 & $8.7 \pm 5.5$ & & $17.9 \pm 5.1$ & & $9.2 \pm 3.9$ & \\
\hline P< & & $0.04^{*}$ & & 0.07 & & 0.81 \\
\hline
\end{tabular}

Table 4: Comparison of the time to achieve the minimum effective plasma concentration (Cper) of valproic acid $(20 \mathrm{ug} / \mathrm{mL}$ defined arbitrary as $20 \mathrm{ug} / \mathrm{mL}$, see text), the time in which the plasma concentrations decay below Cper, and the total duration above Cper plasma concentrations (Tem), during the 24 hour period of observation after the single dose intake of two enteric coated tablets of $500 \mathrm{mg}$ sodium divalproate, by 24 healthy men participating in a cross-over study. All figures are given in hours (mean and SD; ${ }^{*}$ significant).

controlled conditions, there are inter- and intra-individual variations as expected. Figure 2 shows the duration of plasma valproic acid above $\mathrm{C}_{\text {per, }}$ and more than 2 hour differences can be seen in half of the subjects under study. The statistical comparison between both formulations does not show a significant difference when measuring plasma valproic acid levels above $\mathrm{C}_{\mathrm{per}}(\mathrm{p}=0.81)$ within the first 24 hours, after single doses. This is consistent with the equivalence proved in the whole AUCs. Nevertheless, when receiving the test preparation participants show reaching the $\mathrm{C}_{\text {per }}$ value in little more than two hours faster in average, as a result of the statistical influence of 9 of the 24 (37.5\%) participants, which only occurs faster in $12.5 \%(\mathrm{n}=3 / 24)$ for the reference product $(\mathrm{p}<0.04$, significant). In a similar proportion, the maintenance of valproic acid levels above $C_{\text {per }}$ decays in average a couple of hours faster also, so the total effect maintains the equivalence $(\mathrm{p}<0.07$, not significant in this sample) and this data is consistent with the greater variance of $\mathrm{T}_{\text {max }}$ seen before with the reference product.

No adverse events defined as serious were reported during the course of the study, and the incidence of adverse events considered non-serious was similar for both test and reference product, so the investigator could not relate these adverse events with the products received beyond the degree of probability.

\section{Discussion}

Generics of products like divalproate, whose pharmacokinetic is variable [7-12] and its clinical efficacy can be quickly perceived by the prescriber, sometimes create concern in daily practice. For example, some patients switched from the reference compound to a 
Citation: Nunez DA, Schiaffino S, Roldán EJA (2013) Comparison of Two Formulations of Sodium Divalproate Plasma Concentrations after a Single $500 \mathrm{mg}$ Oral Dose in Healthy Subjects, and Stochastic Sub-analysis of the Individual "Clinical Perceptible" Levels. J Bioequiv Availab 5 : 197-200. doi:10.4172/jbb.1000158

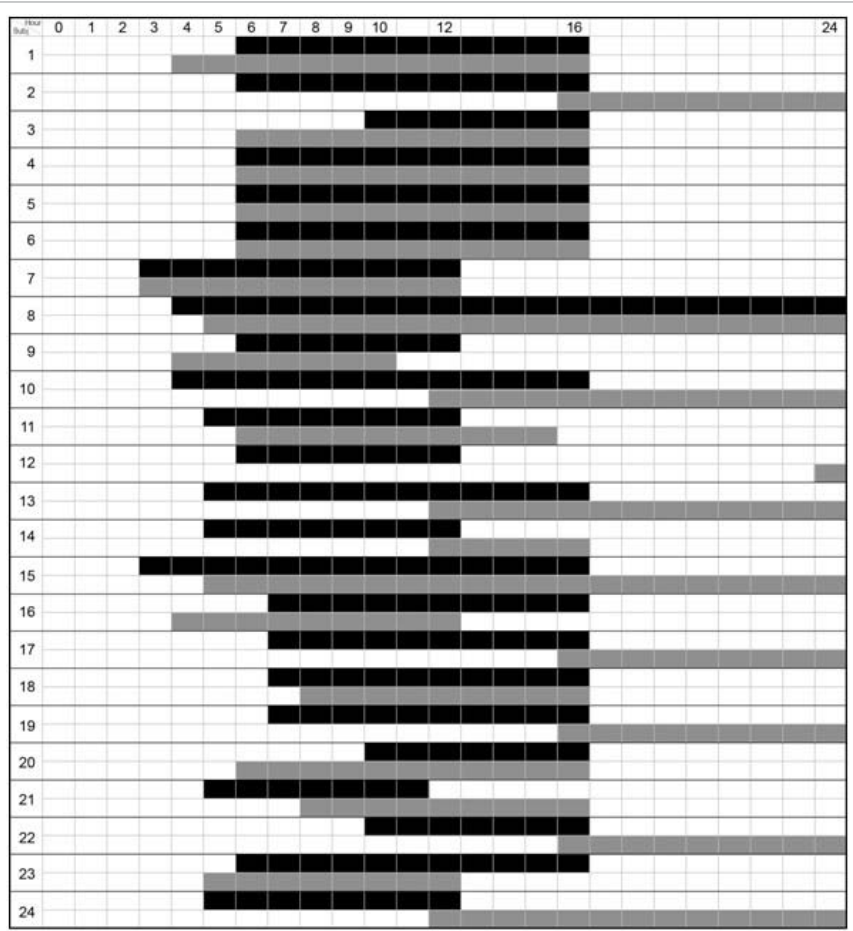

Figure 2: Histogram shows the duration of plasma valproic acid levels above $20 \mu \mathrm{g} / \mathrm{mL}$ (defined as a given effective plasma concentration) during the 24 hour follow up after the single dose intake of a test (black) or reference (grey) enteric coated tablets of $500 \mathrm{mg}$ sodium divalproate, by 24 healthy men participating in a cross -over study.

different formulation are likely to experience minor response due to pharmacokinetics variations. Therefore the prescriber will probably decide to stop the generic even though it had proved to be bioequivalent. Same type of kinetics variations are likely to occur if patients would switch from the generic to the reference form, but such event is unusual in practice; so the wrong belief that generics have an inferior quality may arise. We have noticed that the individual divalproate plasmatic levels and the area under the curve, AUC and $\mathrm{C}_{\max }$ may not differ much intra or inter-individually, but the contour of the curve may vary at the expenses of $\mathrm{T}_{\max }$. That is why we decided, after proving bioequivalence of two formulations by standard procedures, to make a subsequent analysis of the onset and duration of the high portion of the curve of divalproate plasma levels (Figure 2), which are the levels that may possibly produce effects perceived by the practitioner and, as said show variable shapes. In this study, $\mathrm{AUC}_{\text {last }}$ and $\mathrm{C}_{\max }$ were defined as the parameters so as to be able to show bioequivalence between the two products under study. In these conditions results show that geometric mean rates, with a confidence interval of $90 \%$, showed bioequivalence between both products. Then for the subsequent analysis, the $20 \mu \mathrm{g} / \mathrm{mL}$ value of divalproate plasma levels has been chosen as the lower limit of a given stochastic perceived (clinical effective) concentration $\left(\mathrm{C}_{\mathrm{per}}\right)$, based on literature data available. No significant differences in total duration of $\mathrm{C}_{\text {per }}$ were detected in the study, although the test product, due to intra-treatment influences in some subjects, shows a lower mean time in the onset of $\mathrm{C}_{\text {per }}$ after the intake.

\section{Acknowledgments}

This study was sponsored by Gador SA, Buenos Aires (grant 2011-2). The bioequivalence study and the bioequivalence analysis were carried out in Novocap $\mathrm{SA}$. The subsequent intra-treatment variations analysis was carried out in the Scientific Direction of Gador SA.

\section{References}

1. Perucca E (2002) Pharmacological and therapeutic properties of valproate: a summary after 35 years of clinical experience. CNS Drugs 16: 695-714.

2. Brouwer OF, Pieters MS, Edelbroek PM, Bakker AM, van Geel AA et al. (1992) Conventional and controlled release valproate in children with epilepsy: a cross-over study comparing plasma levels and cognitive performances. Epilepsy Res 13: 245-253.

3. Ghose K (1999) Efficacy and safety of long-term sodium valproate therapy in the prophylaxis of migraine headache. Headache Q 10: 127-130.

4. Sherr JD, Kelly DL (1998) Substitution of immediate-release valproic acid for divalproex sodium for adult psychiatric inpatients. Psychiatr Serv 49: 1355 1357.

5. Smith LA, Cornelius VR, Azorin JM, Perugi G, Vieta E, et al. (2010) Valproate for the treatment of acute bipolar depression: systematic review and metaanalysis. J Affect Disord 122: 1-9.

6. Liu MJ, Brouwer KL, Pollack GM (1992) Pharmacokinetics and pharmacodynamics of valproate analogs in rats. III. Pharmacokinetics of valproic acid, cyclohexanecarboxylic acid, and 1-methyl-1-cyclohexanecarboxylic acid in the bile-exteriorized rat. Drug Metab Dispos 20: 810-815.

7. Yamada M, Welty TE (2011) Generic substitution of antiepileptic drugs: a systematic review of prospective and retrospective studies. Ann Pharmacother 45: $1406-1415$.

8. Gidal BE, Tomson T (2008) Debate: Substitution of generic drugs in epilepsy: is there cause for concern? Epilepsia 49 Suppl 9: 56-62.

9. Wangeman M, Retzow A, Vens-Capell B (1999) Pharmacokinetics characteristics of a new multiple unit sustained release formulation of sodium valproate. Int J Clin Pharmacol Ther 37: 100-108.

10. Leppik IE, Hovinga CA (2013) Extended-release antiepileptic drugs: a comparison of pharmacokinetic parameters relative to original immediaterelease formulations. Epilepsia 54: 28-35.

11. Gutiérrez-Gutiérrez G, Poza-Aldea JJ Tellería-Elmezábal J. Martí-Masso JF (2006) Estudio retrospectivos comparando dos formulaciones de ácido valproico.Rev Neurol 42: 129-32.

12. Labiner DM, Paradis PE, Manjunath R, Duh MS, Lafeuille MH, et al. (2010) Generic antiepileptic drugs and associated medical resource utilization in the United States. Neurology 74: 1566-1574. 